\title{
Research Article The Optimal Dispatch of Traffic and Patrol Police Service Platforms
}

\author{
Ke Quan Zhao, ${ }^{1}$ Li Luo, $^{2}$ and Yuan Mei Xia ${ }^{1}$ \\ ${ }^{1}$ College of Mathematics Science, Chongqing Normal University, Chongqing 400047, China \\ ${ }^{2}$ Service Management Institute of Business School, Sichuan University, Chengdu 610064, China \\ Correspondence should be addressed to Li Luo, luolicc@163.com
}

Received 21 September 2012; Accepted 7 November 2012

Academic Editor: Jian-Wen Peng

Copyright (C) 2012 Ke Quan Zhao et al. This is an open access article distributed under the Creative Commons Attribution License, which permits unrestricted use, distribution, and reproduction in any medium, provided the original work is properly cited.

The main goal of this paper is to present a minmax programming model for the optimal dispatch of Traffic and Patrol Police Service Platforms with single traffic congestion. The objective is to minimize the longest time of the dispatch for Traffic and Patrol Police Service Platforms. Some numerical experiments are carried out, and the optimal project is given.

\section{Introduction}

Traffic and Patrol Police Service Platforms (in short, TPPSP) in the city have been playing an important role in dealing with emergency and traffic administration. The national college mathematical modeling contest of China in 2011 proposed the problem related to the optimal dispatch of TPPSP. However, only the case without any traffic congestion is considered for the problem. It is well known that the optimal dispatch and design of TPPSP is very complicated and it is affected by many real factors, such as

(i) the influence of traffic congestion on the optimal dispatch;

(ii) the influence of police resources allocation for each platform;

(iii) the influence of the uncertainty of road weights.

The shortest path between any two nodes in urban traffic network is usually solved by Floyd shortest path algorithm in traffic computing and path search. Also the shortest path algorithms are widely applied to computer science, operational research, geographic information systems and traffic guidance, navigation systems, and so forth [1-5]. Especially, given a detailed GIS mapping and image display program, Liao and Zhong [4] proved that 
the Floyd shortest path algorithm can quickly and easily retrieve the shortest path between two locations, saving computing time and overhead.

The minmax programming model has received more attentions in operations research and optimization fields in the literatures [6-9]. Averbakh and Berman [8] considered the location minmax $p$-TSP problem, where only optimal locations of the servers must be found, without the corresponding tours and without the optimal value of the objective function. Exact linear time algorithms for the cases $p=2$ and $p=3$ are presented.

In recent years, the research on optimal dispatch of TPPSP has also received some attentions in the literatures [10-12]. However, we noted that these works only focused on the case without any traffic congestion for the optimal dispatch of TPPSP.

In this paper, we first consider the optimal dispatch with single traffic congestion when the emergent event and establish a minmax programming model (model II) which objective is to minimize the longest time of the dispatch for TPPSP. Furthermore, some numerical experiments are carried out, and the optimal project is presented.

\section{Notations}

$m$ : The number of the TPPSP

$n$ : The number of intersections that should be blockaded

$d_{i j}^{\prime}:(i=1,2, \ldots, m, j=1,2, \ldots, n)$ The shortest distance from the $i$ th TPPSP to the $j$ th intersection without traffic congestion

$t_{i j}:(i=1,2, \ldots, m, j=1,2, \ldots, n)$ The shortest time from the $i$ th TPPSP to the $j$ th intersection

$v$ : The speed of police vehicles

$x_{i j}:(i=1,2, \ldots, m, j=1,2, \ldots, n)$ The $i$ th TPPSP is dispatched to the $j$ th intersection or not.

Assume that every TPPSP has almost the same police force, TPPSP have been settled at some traffic centers and key parts of an urban area of a city. The average of the police car is $60 \mathrm{~km} / \mathrm{h}$.

\section{Mathematical Models}

In this section, we first introduce a minimax programming model for the optimal dispatch of TPPSP without any traffic congestion in the literatures. Then, we present our main model for the optimal dispatch of TPPSP with single traffic congestion.

\subsection{The Case without Any Traffic Congestion}

When the road section has no traffic congestion, some authors presented the following minimax programming model, which is also a $0-1$ integer programming model, see literatures $[10,12]$, and so forth. 
(model I)

$$
\begin{array}{ll}
\min \max \left\{t_{i j} x_{i j}\right\} & \\
\text { s.t. } & \sum_{i=1}^{m} x_{i j}=1, \quad j=1,2, \ldots, n ; \\
& \sum_{j=1}^{n} x_{i j} \leq 1, \quad i=1,2, \ldots, m ; \\
& x_{i j}\left(1-x_{i j}\right)=0, \quad i=1,2, \ldots, m, j=1,2, \ldots, n ; \\
& t_{i j}=\frac{100 d_{i j}^{\prime}}{60 v}, \quad i=1,2, \ldots, m, j=1,2, \ldots, n .
\end{array}
$$

The objective function (3.1) requires that the maximum time from the $i$ th TPPSP to the $j$ th node which is minimum. Besides, constraint (3.2) requires that every intersection should be blockaded by only one TPPSP. Constraint (3.3) ensures that one TPPSP can only blockade one intersection. Constraint (3.4) requires that $x_{i j}$ is $0-1$ variable. Constraint (3.5) shows that the relation between the time and the distance from the $i$ th TPPSP to the $j$ th intersection. Furthermore, in Constraint (3.5), the unite of $t_{i j}$ is minutes, $v$ is meters per second and the symbol $d_{i j}^{\prime}$ (the unite is millimeters) is the distance of map, $100 d_{i j}^{\prime}$ (the unite is meters) is the real distance.

\subsection{The Case with Single Traffic Congestion}

In the real life, traffic congestion may occur in urban traffic network. Therefore, the research on the optimal dispatch of TPPSP with Traffic congestions is important and meaningful. Considering that the emergency may occur at any time and place, and the road section may have some traffic congestions, in this subsection, we present one minmax programming model for the optimal dispatch of TPPSP with single traffic congestion. The optimal dispatch of TPPSP with Traffic congestions model is more effective than model I. Moreover, the optimal dispatch of TPPSP with Traffic congestions model's results has immediate practical applications.

We assume that the traffic congestion occurs on the road section from the node $p$ to the node $q$, where the node $p$ is adjacent to the node $q$. And $T_{p q}$ denotes the average time of blocking. Besides, $d_{i j}^{\prime \prime}$ denotes the shortest distance from the TPPSP $i$ to the $j$ th intersection without the road section from the node $p$ to the node $q, P_{i j}$ denotes the shortest path from the $i$ th TPPSP to the $j$ th node. $R$ denotes the set of $P_{i j}$ which go through $p$ and $q$ nodes. $t_{i j}^{\prime}(i=1,2, \ldots, m, j=1,2, \ldots, n)$ denotes the shortest time from the $i$ th TPPSP to the $j$ th intersection without traffic congestion, and $t_{i j}^{\prime \prime}$ denotes the shortest time from the $i$ th TPPSP to the $j$ th intersection without the road section from the node $p$ to the node $q$.

We establish the following minmax programming model II for the dispatch of TPPSP with single traffic congestion: 
(model II)

$$
\begin{array}{ll}
\min & \max \left\{t_{i j} x_{i j}\right\} \\
\text { s.t. } & \sum_{i=1}^{m} x_{i j}=1, \quad j=1,2, \ldots, n ; \\
& \sum_{j=1}^{n} x_{i j} \leq 1, \quad i=1,2, \ldots, m ; \\
& x_{i j}\left(1-x_{i j}\right)=0, \quad i=1,2, \ldots, m, j=1,2, \ldots, n,
\end{array}
$$

where

$$
\begin{gathered}
t_{i j}= \begin{cases}t_{i j \prime}^{\prime} & P_{i j} \in A_{1} ; \\
t_{i j}^{\prime \prime} & P_{i j} \in A_{2} ; \\
t_{i j}^{\prime}+T_{p q}-\min \left\{t_{i p^{\prime}}^{\prime} t_{i q}^{\prime}\right\}, & P_{i j} \in A_{3} ;\end{cases} \\
t_{i j}^{\prime}=\frac{100 d_{i j}^{\prime}}{60 v} \quad i=1,2, \ldots, m, j=1,2, \ldots, n ; \\
t_{i j}^{\prime \prime}=\frac{100 d_{i j}^{\prime \prime}}{60 v} \quad i=1,2, \ldots, m, j=1,2, \ldots, n ; \\
A_{1}=\left\{P_{i j} \mid P_{i j} \notin R \text { or } P_{i j} \in R, \min \left\{t_{i p}^{\prime}, t_{i q}^{\prime}\right\} \geq T_{p q}\right\} ; \\
A_{3}=\left\{P_{i j} \mid P_{i j} \in R, \min \left\{t_{i p}^{\prime}, t_{i q}^{\prime}\right\}<T_{p q}, t_{i j}^{\prime}+T_{p q}-\min \left\{t_{i p}^{\prime}, t_{i q}^{\prime}\right\}>t_{i j}^{\prime \prime}\right\} ; \\
\text { a } \left.P_{i j} \mid P_{i j} \in R, \min \left\{t_{i p}^{\prime}, t_{i q}^{\prime}\right\}<T_{p q}, t_{i j}^{\prime}+T_{p q}-\min \left\{t_{i p}^{\prime}, t_{i q}^{\prime}\right\} \leq t_{i j}^{\prime \prime}\right\} .
\end{gathered}
$$

The objective function (3.6) requires that the maximum times from the $i$ th TPPSP to the $j$ th node which is minimum. The analysis of constraints (3.6)-(3.9) is the same as constraints (3.2)-(3.4). However, the value of $t_{i j}$ is different from the time of the model I. In the model II, the function of $t_{i j}$ is divided into three segments.

\section{Numerical Experiments}

In this paper, we take $m=20, n=|I|=13$, where

$$
I=\{12,14,16,21,22,23,24,28,29,30,38,48,62\}
$$

to do specific analysis for our model. The data is based on (http://www.mcm.edu.cn/). We use Floyd Shortest Path Algorithm to figure out $d_{i j}^{\prime}$ and $d_{i j}^{\prime \prime}$ by Matlab software. We can 
Table 1: The optimal dispatch project of TPPSP without any traffic congestion.

\begin{tabular}{lcc}
\hline Dispatching project & Path of choosing project & $\begin{array}{c}\text { Time from the } i \text { th TPPSP } \\
\text { to the } j \text { th intersection }\end{array}$ \\
\hline $1-38$ & $1-69-70-2-40-39-38$ & 5.880900 \\
$2-16$ & $2-40-39-38-16$ & 7.388100 \\
$4-48$ & $4-57-58-59-51-50-5-47-48$ & 7.395900 \\
$7-29$ & $7-30-29$ & 8.015500 \\
$9-30$ & $9-34-33-32-7-30$ & 3.492300 \\
$10-12$ & $10-26-27-12$ & 7.586600 \\
$11-22$ & $11-22$ & 3.269600 \\
$12-23$ & $12-25-24-13-23$ & 6.477000 \\
$13-24$ & $13-24$ & 2.385400 \\
$14-21$ & $14-21$ & 3.265000 \\
$15-28$ & $15-28$ & 4.751800 \\
$16-14$ & $16-14$ & 6.741700 \\
$20-62$ & $20-85-62$ & 6.448900 \\
\hline
\end{tabular}

Table 2: The optimal dispatch project of TPPSP with $T_{p q}=5 \mathrm{~min}$.

\begin{tabular}{lcc}
\hline Dispatching project & Path of choosing project & $\begin{array}{c}\text { Time from the } i \text { th TPPSP } \\
\text { to the } j \text { th intersection }\end{array}$ \\
\hline $1-38$ & $1-69-70-2-40-39-38$ & 5.880900 \\
$2-16$ & $2-40-39-38-16$ & 7.388100 \\
$4-48$ & $4-57-58-59-51-50-5-47-48$ & 7.395900 \\
$5-30$ & $5-47-48-30$ & 3.182900 \\
$7-29$ & $7-30-29$ & 8.015500 \\
$10-12$ & $10-26-27-12$ & 7.586600 \\
$11-24$ & $11-25-24$ & 3.805300 \\
$12-22$ & $12-25-24-13-22$ & 6.882500 \\
$13-23$ & $13-23$ & 0.500000 \\
$14-21$ & $14-21$ & 3.265000 \\
$15-28$ & $15-28$ & 4.751800 \\
$16-14$ & $16-14$ & 6.741700 \\
$18-62$ & $18-80-79-19-77-76-64-63-4-62$ & 6.734400 \\
\hline
\end{tabular}

obtain the dispatch project of TPPSP without any traffic congestion when the emergent event happens in the city as Table 1.

Considering the case with single traffic congestion for the optimal dispatch of TPPSP in urban traffic network, we do the numerical experiments for the minmax programming model II by using Matlab software. Here, we take $p=36, q=16, T_{p q}=5 \mathrm{~min}$. The dispatching project of TPPSP with one road section having single traffic congestion when the emergent event happens is shown in Table 2. 
Table 3: The optimal dispatch project of TPPSP with $T_{p q}=10 \mathrm{~min}$.

\begin{tabular}{lcc}
\hline Dispatching project & Path of choosing project & $\begin{array}{c}\text { Time from the } i \text { th TPPSP } \\
\text { to the } j \text { th intersection }\end{array}$ \\
\hline $1-38$ & $1-69-70-2-40-39-38$ & 5.880900 \\
$2-16$ & $2-40-39-38-16$ & 7.388100 \\
$5-62$ & $5-50-51-59-58-57-60-62$ & 5.255100 \\
$6-48$ & $6-47-48$ & 2.506400 \\
$7-29$ & $7-30-29$ & 8.015500 \\
$8-30$ & $8-33-32-7-30$ & 3.060800 \\
$10-12$ & $10-26-27-12$ & 7.586600 \\
$11-23$ & $11-22-13-23$ & 4.675100 \\
$12-22$ & $12-25-24-13-22$ & 6.882500 \\
$13-24$ & $13-24$ & 2.385400 \\
$14-21$ & $14-21$ & 3.265000 \\
$15-28$ & $15-28$ & 4.751800 \\
$16-14$ & $16-14$ & 6.741700 \\
\hline
\end{tabular}

Table 4: The optimal dispatch project of TPPSP with $T_{p q}=30 \mathrm{~min}$.

\begin{tabular}{lcc}
\hline Dispatching project & Path of choosing project & $\begin{array}{c}\text { Time from the } i \text { th TPPSP } \\
\text { to the } j \text { th intersection }\end{array}$ \\
\hline $1-62$ & $1-75-76-64-63-4-62$ & 4.885200 \\
$2-16$ & $2-40-39-38-16$ & 7.388100 \\
$6-30$ & $6-47-48-30$ & 3.213500 \\
$7-29$ & $7-30-29$ & 8.015500 \\
$8-48$ & $8-47-48$ & 3.099500 \\
$9-38$ & $9-35-36-39-38$ & 4.725700 \\
$10-22$ & $10-26-11-22$ & 7.707900 \\
$11-23$ & $11-22-13-23$ & 4.675100 \\
$12-12$ & $12-12$ & 0.000000 \\
$13-24$ & $13-24$ & 2.385400 \\
$14-21$ & $14-21$ & 3.265000 \\
$15-28$ & $15-28$ & 4.751800 \\
$16-14$ & $16-14$ & 6.741700 \\
\hline
\end{tabular}

From Tables 1 and 2, we can clearly see that the maximum time of the optimal dispatch for TPPSP is the same for the case without any traffic congestion and the case with single traffic congestion in the given urban traffic network. However, the optimal dispatch project of TPPSP is different each other. Consequently, this shows that traffic congestion between the nodes in urban traffic network system will influence the optimal dispatch project of TPPSP in a certain degree when the emergent event happens. 
Table 5: The optimal dispatch project of TPPSP with $T_{p q}=60 \mathrm{~min}$.

\begin{tabular}{lcc}
\hline Dispatching project & Path of choosing project & $\begin{array}{c}\text { Time from the } i \text { th TPPSP } \\
\text { to the } j \text { th intersection }\end{array}$ \\
\hline $2-16$ & $2-40-39-38-16$ & 7.388100 \\
$4-48$ & $4-57-58-59-51-50-5-47-48$ & 7.395900 \\
$7-29$ & $7-30-29$ & 8.015500 \\
$9-30$ & $9-34-33-32-7-30$ & 3.492300 \\
$10-22$ & $10-26-11-22$ & 7.707900 \\
$11-24$ & $11-25-24$ & 3.805300 \\
$12-23$ & $12-25-24-13-23$ & 6.477000 \\
$13-12$ & $13-24-25-12$ & 5.977000 \\
$14-21$ & $14-21$ & 3.265000 \\
$15-28$ & $15-28$ & 4.751800 \\
$16-14$ & $16-14$ & 6.741700 \\
$19-38$ & $19-79-78-1-69-70-2-40-39-38$ & 7.639300 \\
$20-62$ & $20-85-62$ & 6.448900 \\
\hline
\end{tabular}

From Tables 2, 3, 4, and 5, we can gain the different dispatch project when the time of a traffic congestions is different. We can know the traffic congestion can influence the dispatching project. The influence degree is different when the time of a traffic congestion is different. However, for the node $p$ and node $q$, the maximum time from the TPPSP to the intersection is $8.015500 \mathrm{~min}$ when the time of a traffic congestion is different.

In order to avoid the data that we use may be too special, we further take $p=7, q=30$. Still take $m=20, n=13$, and $T_{p q}=5$ or 10 or 30 or $60 \mathrm{~min}$, respectively, the dispatching project about the TPPSP with one road section having a traffic congestion when the emergent event happens as Table 6, where $M_{1}$ means blocking time, $M_{2}$ means dispatching project, $M_{3}$ means path of choosing project, and $M_{4}$ means time from the $i$ th TPPSP to the $j$ th intersection.

In Table 6, where $p=7, q=30$, we can also gain the different dispatching project when the time of a traffic congestion is different. However, the maximum time from the TPPSP to the intersection is $8.570200 \mathrm{~min}$ when the time of a traffic congestion is different.

Road section with having a traffic congestion is different, the maximum time from the TPPSP to the intersection is different. The influence degree of the time of a traffic congestion is not too large to the maximum time from the TPPSP to the intersection, but is large to the dispatching project.

\section{Concluding Remarks}

In this paper, we present a minmax programming models for the optimal dispatch of TPPSP with single traffic congestion. Some numerical experiments are carried out by using Matlab software and the optimal dispatch projects are given. However, in this paper, we only consider the case with single traffic congestion in model II. Hence, it is possible and meaningful to study the optimal dispatch project of TPPSP with several traffic congestions. This will be the future topics that we study. 
Table 6: The optimal dispatch project of TPPSP with $T_{p q}=5,10,30,60 \mathrm{~min}$.

\begin{tabular}{|c|c|c|c|c|}
\hline$M_{1}$ & $5 \mathrm{~min}$ & $10 \mathrm{~min}$ & $30 \mathrm{~min}$ & $60 \mathrm{~min}$ \\
\hline \multirow{13}{*}{$M_{2}$} & $1 \rightarrow 38$ & $1 \rightarrow 38$ & $1 \rightarrow 38$ & $3 \rightarrow 48$ \\
\hline & $4 \rightarrow 48$ & $5 \rightarrow 48$ & $2 \rightarrow 16$ & $7 \rightarrow 28$ \\
\hline & $5 \rightarrow 16$ & $7 \rightarrow 28$ & $4 \rightarrow 48$ & $8 \rightarrow 30$ \\
\hline & $6 \rightarrow 62$ & $9 \rightarrow 14$ & $7 \rightarrow 28$ & $10 \rightarrow 24$ \\
\hline & $7 \rightarrow 28$ & $10 \rightarrow 12$ & $9 \rightarrow 14$ & $11 \rightarrow 21$ \\
\hline & $9 \rightarrow 14$ & $11 \rightarrow 21$ & $10 \rightarrow 22$ & $12 \rightarrow 21$ \\
\hline & $10 \rightarrow 22$ & $12 \rightarrow 22$ & $11 \rightarrow 12$ & $13 \rightarrow 22$ \\
\hline & $11 \rightarrow 23$ & $13 \rightarrow 24$ & $12 \rightarrow 24$ & $14 \rightarrow 23$ \\
\hline & $12 \rightarrow 24$ & $14 \rightarrow 23$ & $13 \rightarrow 23$ & $15 \rightarrow 29$ \\
\hline & $13 \rightarrow 12$ & $15 \rightarrow 29$ & $14 \rightarrow 21$ & $16 \rightarrow 14$ \\
\hline & $14 \rightarrow 21$ & $16 \rightarrow 30$ & $15 \rightarrow 29$ & $17 \rightarrow 16$ \\
\hline & $15 \rightarrow 29$ & $17 \rightarrow 16$ & $16 \rightarrow 30$ & $19 \rightarrow 38$ \\
\hline & $16 \rightarrow 30$ & $20 \rightarrow 62$ & $20 \rightarrow 62$ & $20 \rightarrow 62$ \\
\hline \multirow{13}{*}{$M_{3}$} & 1-69-70-2-40-39-38 & $1-69-70-2-40-39-38$ & $1-69-70-2-40-39-38$ & $3-55-54-53-49-5-47-48$ \\
\hline & $-57-58-59-51-50-5-47-48$ & $5-47-48$ & $2-40-39-38-16$ & $7-15-28$ \\
\hline & $5-47-8-9-35-36-16$ & $7-15-28$ & $4-57-58-59-51-50-5-47-48$ & $8-47-48-30$ \\
\hline & $6-59-58-57-60-62$ & $9-35-36-16-14$ & $7-15-28$ & $10-26-11-25-24$ \\
\hline & $7-15-28$ & $10-26-27-12$ & $9-35-36-16-14$ & $11-22-21$ \\
\hline & 9-35-36-16-14 & $11-22-21$ & $10-26-11-22$ & $12-12$ \\
\hline & $10-26-11-22$ & $12-25-24-13-22$ & $11-25-12$ & $13-22$ \\
\hline & $11-22-13-23$ & $13-24$ & $12-25-24$ & $14-21-22-13-23$ \\
\hline & $12-25-24$ & $14-21-22-13-23$ & $13-23$ & $15-28-29$ \\
\hline & $13-24-25-12$ & $15-28-29$ & $14-21$ & $16-14$ \\
\hline & $14-21$ & $16-36-35-9-8-47-48-30$ & $15-28-29$ & $17-40-39-38-16$ \\
\hline & $15-28-29$ & $17-40-39-38-16$ & $16-36-35-9-8-47-48-30$ & $19-79-78-1-69-70-2-40-39-38$ \\
\hline & $16-36-37-7-30$ & $20-85-62$ & 20-85-62 & $20-85-62$ \\
\hline \multirow{13}{*}{$M_{4}$} & 5.880900 & 5.880900 & 5.880900 & 8.197900 \\
\hline & 7.395900 & 2.475800 & 7.388100 & 8.570200 \\
\hline & 6.228000 & 8.570200 & 7.395900 & 3.806600 \\
\hline & 5.337300 & 8.274200 & 8.570200 & 8.243600 \\
\hline & 8.570200 & 7.586600 & 8.274200 & 5.072300 \\
\hline & 8.274200 & 5.072300 & 7.707900 & 0.000000 \\
\hline & 7.707900 & 6.882500 & 3.791400 & 0.905540 \\
\hline & 4.675100 & 2.385400 & 3.591600 & 6.473300 \\
\hline & 3.591600 & 6.473300 & 0.500000 & 5.700500 \\
\hline & 5.977000 & 5.700500 & 3.265000 & 6.741700 \\
\hline & 3.265000 & 6.498900 & 5.700500 & 8.161600 \\
\hline & 5.700500 & 8.161600 & 6.498900 & 7.639300 \\
\hline & 5.583100 & 6.448900 & 6.448900 & 6.448900 \\
\hline
\end{tabular}

\section{Acknowledgments}

This work is partially supported by the National Natural Science Foundation of China (Grants 71131006, 71172197, 11171363, 11271391, 11001289), Central University Fund of Sichuan University under Grant no. skgt201202, the Special Fund of Chongqing 
Key Laboratory (CSTC, 2011KLORSE02), the Natural Science Foundation Project of Chongqing (Grant CSTS2012jjA00002), and the Education Committee Research Foundation of Chongqing (Grant KJ110625).

\section{References}

[1] T. Dudás and R. Rudolf, "Spanning trees and shortest paths in monge graphs," Computing, vol. 60, no. 2, pp. 109-119, 1998.

[2] S. Bistarelli, U. Montanari, and F. Rossi, "Soft constraint logic programming and generalized shortest path problems," Journal of Heuristics, vol. 8, no. 1, pp. 25-41, 2002.

[3] B. Huang, W. M. Wu, and Y. N. Zheng, "The research and improvement of path optimization in vehicle navigation," Advances in Intelligent and Soft Computing, vol. 129, pp. 203-207, 2012.

[4] Y. M. Liao and J. Zhong, "FLOYD Algorithm Based on the Shortest Path in GIS," Communications in Computerand Information Science, vol. 267, no. 4, pp. 574-579, 2012.

[5] H. X. Li, Q. J. Xu, and D. Q. Zhang, "An efficient constrained shortest path algorithm for traffic navigation," Advanced Materials Research, vol. 356-360, pp. 2880-2885, 2012.

[6] K. Fan, "Minimax theorems," Proceedings of the National Academy of Sciences of the United States of America, vol. 39, pp. 42-47, 1953.

[7] G. Mosheiov and M. Shadmon, "Minmax earliness-tardiness costs with unit processing time jobs," European Journal of Operational Research, vol. 130, no. 3, pp. 638-652, 2001.

[8] I. Averbakh and O. Berman, "Minmax p-traveling salesmen location problems on a tree," Annals of Operations Research, vol. 110, no. 1-4, pp. 55-68, 2002.

[9] G. Mosheiov and D. Oron, "Minmax scheduling with job-classes and earliness-tardiness costs," European Journal of Operational Research, vol. 177, no. 1, pp. 612-622, 2007.

[10] J. X. Yu, D. C. Song, X. Y. Zhao, and J. Q. Li, “Research on the reasonable dispatch of service platform of trafficand patrol police," Science Technology and Engineering, vol. 12, pp. 126-128, 2012.

[11] S. L. Fu, L. Fang, C. L. Wang, and X. W. Yang, "The establishment and scheduling of traffic and patrol police service platform," Journal of Logistical Engineering Universtiy, vol. 28, pp. 79-84, 2012.

[12] X. Wei, X. X. Dong, and D. Tang, "The setting and dispatch model of the traffic and cruising police platform," Mathematical Modeling and Its Applications, vol. 1, pp. 73-76, 2012. 


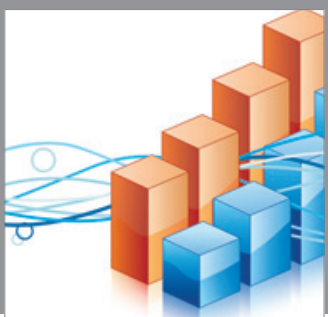

Advances in

Operations Research

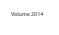

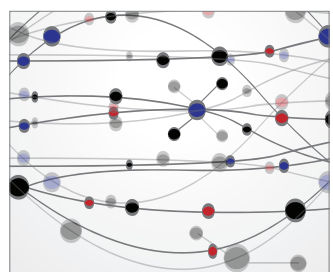

\section{The Scientific} World Journal
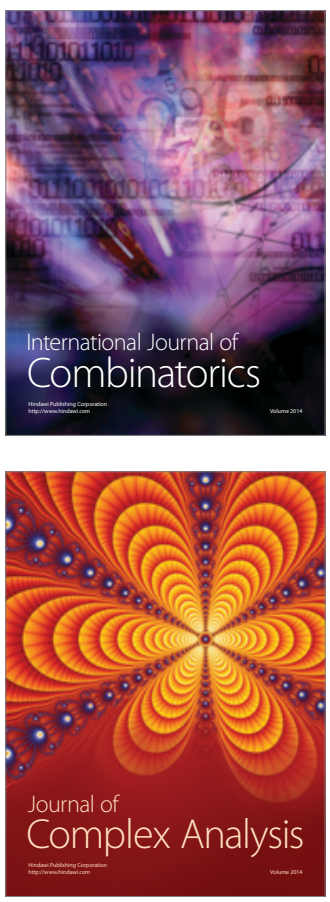

International Journal of

Mathematics and

Mathematical

Sciences
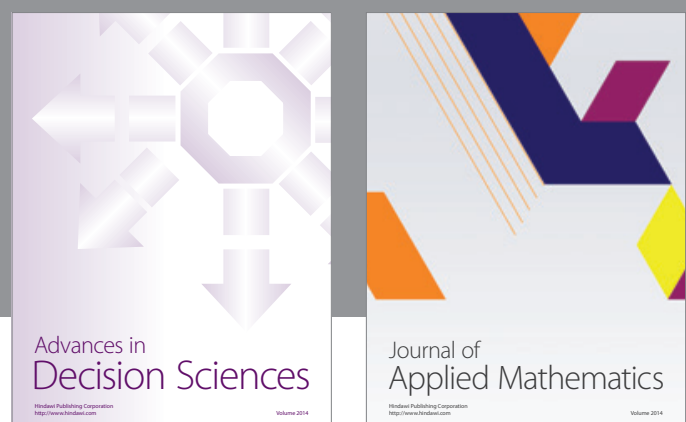

Journal of

Applied Mathematics
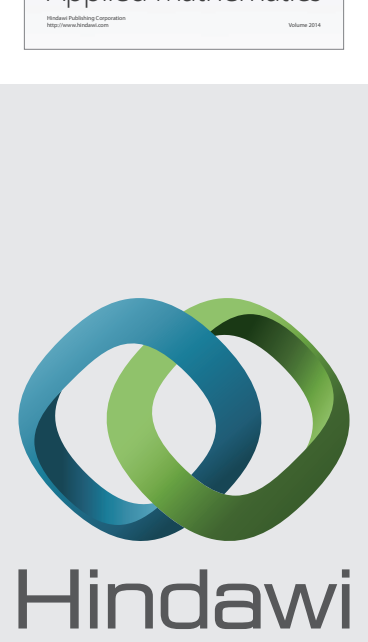

Submit your manuscripts at http://www.hindawi.com
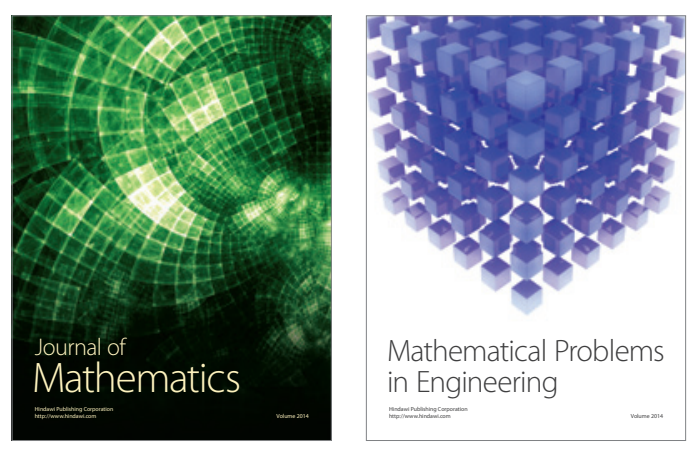

Mathematical Problems in Engineering
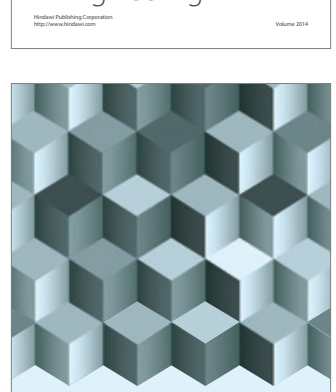

Journal of

Function Spaces
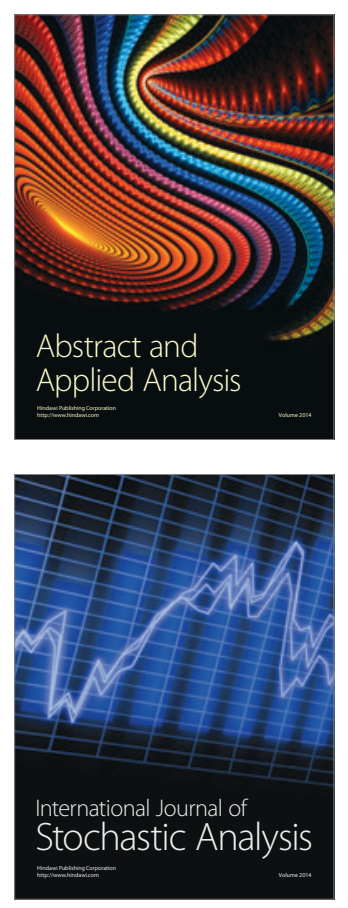

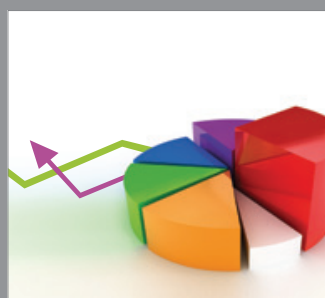

ournal of

Probability and Statistics

Promensencen
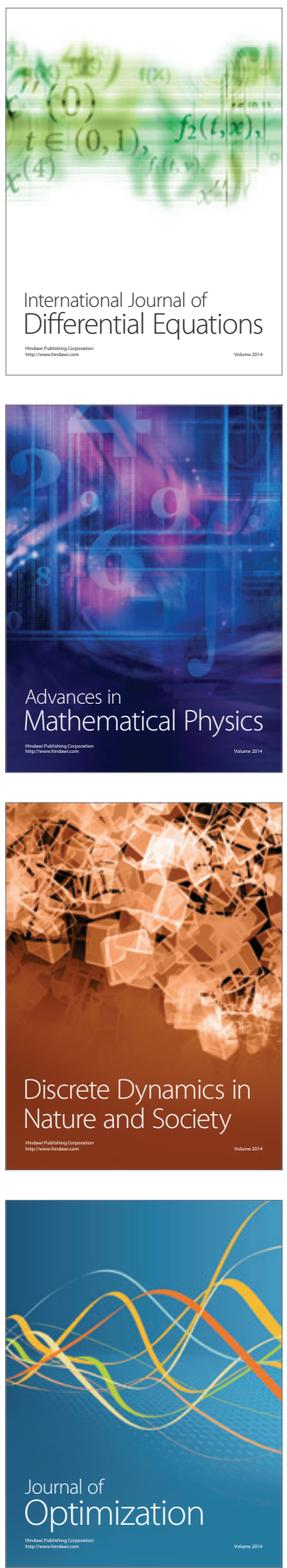